\title{
EVALUACIÓN DE LA REGENERACION DE Acacia decurrens, Acacia melanoxylon Y Ulex europaeus EN ÁREAS EN PROCESO DE RESTAURACIÓN ECOLÓGICA
}

\author{
JAIRO HERNÁN SOLORZA BEJARANO ${ }^{1}$
}

Manizales, 2011-12-05 (Rev. 2012-05-27)

\section{RESUMEN}

Se evaluó la regeneración de Acacia decurrens, Acacia melanoxylon y Ulex europaeus, en una zona sujeta a un proceso de restauaración ecológica. En las áreas con presencia de individuos arboreos de Acacia decurrens y Acacia melanoxylon se presenta expresión del banco de semillas con formación periódica de la capa de hojarasca que detiene o desvía el proceso sucesional. En las áreas de claro se presenta regeneración de Ulex europaeus, ocupando rápidamente los espacios libres de vegetación. La plantación de especies nativas de porte arbustivo y arbóreo, junto con el control periódico de la regeneración y formación de hojarasca de las especies tensionantes, facilita la activación de los mecanismos de sucesión secundaria, aumentando la riqueza, diversidad y equitatividad de las especies en el ecosistema.

\section{PALABRAS CLAVE:}

Acacia decurrens, Acacia melanoxylon, Ulex europaeus, regeneración, restauración ecológica, sucesión secundaria.

\section{EVALUATION OF REGENERATION OF Acacia decurrens, Acacia melanoxylon AND Ulex europaeus IN AREAS IN PROCESS OF ECOLOGICAL RESTORATION}

\begin{abstract}
Regeneration of Acacia decurrens, Acacia melanoxylon and Ulex europaeus In an area subject to the process of ecological restoration was evaluated. In areas with Acacia decurrens and Acacia melanoxylon arboreal individuals expression of seed banks with periodic accumulation of fallen leaves that stop or divert the succession process is present. In open areas Ulex europaeus regeneration occurs quickly occupying areas without vegetation. The plantation of native shrubby or arboreal species, along with periodic monitoring of the regeneration and fallen leaves formation of alien species, facilitate the activation of secondary succesion mechanisms, increasing the richness, diversity and evenness of species in the ecosystem.
\end{abstract}

\section{KEY WORDS:}

Acacia decurrens, Acacia melanoxylon, Ulex europaeus, ecological restoration, regeneration, secondary successión.

\section{INTRODUCCIÓN}

Los Cerros Orientales de Bogotá, históricamente han sido fuente de bienes y servicios ambientales en los procesos de expansión urbana, que han generado pérdida de la vegetación nativa y la reforestación con especies exóticas como Acacia decurrens, Acacia melanoxylon y 
Ulex europaeus, entre otras, que alteran la estructura (diversidad de fauna y flora, estratificación, cadenas tróficas) y función (ciclos de nutrientes, producción de materia orgánica) de los ecosistemas (DAMA \& BACHAQUEROS, 2000). Generando exclusión e inhibición competitiva de las especies nativas (Mack et al. 2000), aumentando el riesgo de disturbios naturales y antrópicos como los relacionados con procesos erosivos de remoción en masa, eventos pirogenéticos, alteración de los ciclos hidrológicos y modificación de las características físico-químicas del suelo (Rodríguez, 2009).

El género Acacia incluye especies fijadoras de nitrógeno, que generan asociaciones simbióticas con microorganismos del suelo de los géneros Rhizobium y Bradyrhizobium (Quiceno \& Medina, 2006). favoreciendo su adaptación y crecimiento en suelos degradados (Medina et al. 2008; Santana 2007; Leython \& Ruiz 2006) con un incremento en los requerimientos hídricos superiores a las especies nativas (Matthews, 2005), limitando la oferta del recurso en el suelo. Adicionalmente, la producción de semillas es abundante, entre 60.000 y 90.000 semillas $/ \mathrm{Kg}$ (Quiroz, I. et al. 2009) resistente y de alta viabilidad durante largos períodos de tiempo, que se activa en un amplio rango de condiciones de luz y temperatura con escoriación de la testa (Funes et al. 2009; Bañalres et al. s.f.). La constante defoliación y dispersión de semillas va formando gradualmente una capa densa de hojarasca en el suelo que limita la oferta de micrositios de implantanción y acceso al recurso lumínico, cambia las condiciones químicas, generan alelopatía y aumentan la recurrencia de disturbios pirogenéticos (DAMA \& SUNA HISCA, 2002). que favorecen la regeneración de la acacia (García et al. 2010; Gómez, 2008).

Los matorrales invasores de Ulex euroapeus inhiben la colonización y establecimiento de especies nativas por el alto nivel de competencia, colonizando suelos degradados de baja fertilidad o erosionados en espacios abiertos, bordes de quebrada o carreteras (Sánchez, 2009), con raíces profundas que maximizan la absorción de agua y nutrientes, rápida regeneración vegetativa después de disturbios por corte o fuego, coberturas densas de porte arbustivo que no ofrece espacios para el arribo de propágulos de otras especies (Zabaleta \& Vargas 2008; Holmberg et al. 2007). El factor desencadenante de la recurrencia de incendios en los complejos invasores de Ulex europaeus, está ligado a las abundantes cantidades de necromasa en pie y la baja humedad (Ríos, 2010), favoreciendo la regeneración vegetativa y expresión del banco de semillas de la especie invasora (Vargas 2006).

Estas especies tensionantes generan una desviación o detienen el proceso de sucesión secundaria en los ecosistemas zonales (DAMA \& BACHAQUEROS, 2000). Causando exclusión de la flora y fauna nativa, alteraciones edáficas e hidrológicas (Aguilar, 2010; Quiroz, C. et al. 2009). La restauración ecológica, reactiva el proceso de sucesión secundaria (Galvis, 2002). Facilitando los procesos naturales en la dinámica ecológica de los ecosistemas, para generar conectividad con relictos de vegetación conservada (Vargas, 2009).

La evaluación de la dinámica de regeneración de Acacia decurrens, Acacia melanoxylon y Ulex europaeus, en escenarios con diferentes condiciones ecológicas, permite diseñar la estrategia de actuación y manejo adaptativo en el proceso de restauración ecológica, para la activación de los mecanismos sucesionales que devuelvan los atributos estructurales y funcionales del ecosistema, como el aumento de la biodiversidad, conectividad con los relictos de vegetación nativa, dinamización del ciclo de nutrientes, incremento de la productividad, etc.

Esta investigación busca establecer la dinámica de regeneración de Acacia decurrens, Acacia melanoxylon y Ulex europaeus dentro del 
proceso de restauración ecológica adelantado en el Parque Nacional Enrique Olaya Herrera, por el Jardín Botánico José Celestino Mutis.

\section{MÉTODOS}

La investigación se adelantó en Bogotá D.C., Colombia, en una zona de restauración ecológica localizada en el Parque Nacional Enrique Olaya

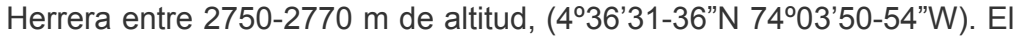
área se caracteriza por presentar un régimen bimodal de lluvias con picos de máxima precipitación entre marzo-mayo y octubre-diciembre y dos picos de menor precipitación en enero-febrero, junio-septiembre, sin temporadas de sequía, con media anual de humedad relativa del $81 \%$ y evaporación menor a $70 \mathrm{~mm}$ mensuales (Datos Estación climatológica IDEAM, Estación Venado de Oro, medias mensuales multianuales 19892008).

El ecosistema degradado corresponde a una reforestación "protectora" de Acacia decurrens (área blanco), que se constituyó en un monocultivo con exclusión de las especies nativas y la formación de la capa de hojarasca incrementando el riesgo de eventos pirogenéticos. Los individuos de acacia dominan el estrato arbustivo y arbóreo con una cobertura de dosel del $80 \%$ y una cobertura de hojarasca del $96 \%$. Hay presencia de individuos aislados de especies nativas en el estrato herbáceo establecidas en el borde del área. El ecosistema se encuentra detenido en una fase sucesional temprana, donde no se presenta regeneración de especies nativas bajo el dosel arbóreo y escasa presencia de fauna (insectos y aves principalmente), limitando los procesos funcionales propios de los ecosistemas zonales, constituyendo un riesgo para la generación y propagación de incendios forestales. Hay presencia de matorrales invasores de Ulex europaeus en los alrededores, sin espacios de suelo descubierto.

El proceso de restauración ecológica adelantado por el Jardín Botánico José Celestino Mutis, consistió en la estabilización superficial del terreno mediante trinchos, para evitar el proceso erosivo continuo y la pérdida de nutrientes, poda al tercio bajo no superior al 30\% del follaje, eliminación total de individuos juveniles de Acacia decurrens y Acacia melanoxylon, corte y eliminación de los individuos de Ulex europaeus además de, remoción de la capa de hojarasca en las áreas reforestadas con acacias. Posteriormente se llevó a cabo repoblamiento vegetal con especies nativas arbustivas y arbóreas (Juglans neotropica, Quercus humboldtii, Myrcianthes leucoxyla, Tibouchina mollis, Aegiphilla bogotensis, Abatia parviflora, Dodonaea viscosa, Ageratina aristeii y Baccharis latifolia) en una densidad de 700 individuos/Ha, en diferentes áreas de la zona intervenida, de acuerdo a la necesidad que representan por la ausencia de especies nativas silvestres de porte arbustivo y arbóreo.

Las especies reintroducidas poseen diferentes rasgos de historia de vida que están relacionadas con especies leñosas precursoras en áreas de rápido crecimiento, con aporte de materia orgánica y producción de propágulos periódicos, que permitan generar corredores para el tránsito de aves y sirvan de hábitat de fauna (artrópodos principalmente) como Dodonaea viscosa, Myrcianthes leucoxyla, Tibouchina mollis, Abatia parviflora, Ageratina aristeii y Baccharis latifolia, especies de árboles nativos dominantes de la estructura a futuro en el área en proceso de restauración ecológica como Aegiphilla bogotensis, Juglans neotropica y Quercus humboldtii. La especies fueron plantadas en un diseño de módulos, con una especie central que corresponde a la de lento 
crecimiento, características de bosques secundarios maduros y un anillo de siete individuos que corresponden a las especies precursoras leñosas de rápido crecimiento, separado entre sí por una distancia de 1,5 metros.

El objetivo es generar una estructura de especies arbustivas o arbóreas de porte bajo que a mediano plazo genere corredores de conectividad, hagan el aporte de materia orgánica al suelo, cambien las condiciones microclimáticas del área (temperatura, humedad) y favorezcan la regeneración y arribo de propágulos de especies nativas silvestres, controle la expresión de las especies tensionantes invasoras y permita el establecimiento además de la persistencia de las especies que dominen la estructura a largo plazo en el ecosistema rehabilitado.

Se seleccionaron tres áreas, cada una de 0,5 $\mathrm{Ha}$, con diferentes características estructurales y fisiográficas fisionómicas, en las cuales se llevaron a cabo las actividades de intervención, para evaluar la regeneración de $A$. decurrens, $A$. melanoxylon, $U$. europaeus, así como la cobertura de hojarasca y suelo descubierto.

El área intervenida 1 , presenta una pendiente entre $10^{\circ}$ y $15^{\circ}$ de inclinación, con una cobertura arbórea del 10\% compuesta por Acacia melanoxylon, matorrales de especies nativas y frentes de avance de matorrales de Ulex europaeus en los alrededores. El mantenimiento se llevó a cabo a los 150 y 180 días, consistente en el rastrillado de la hojarasca, remoción de renuevos de Ulex europaeus y reintroducción de especies nativas (350 individuos/0,5 Ha).

El área intervenida 2, es un terreno con una inclinación menor a $10^{\circ}$, con una cobertura arbustiva y arbórea del $40 \%$ compuesta por Acacia decurrens, Acacia melanoxylon, Quercus humboldtii, Pittosporum undulatum, Miconia squamulosa y Duranta mutissi. El mantenimiento en el área se llevó a cabo a los 30 días, con el rastrillado de la hojarasca, sin plantación de especies nativas.

El área intervenida 3 , presenta una inclinación menor a $10^{\circ}$, con cobertura mixta de especies herbáceas en las que dominan Pteridium aquilinum y Rubus bogotensis, con presencia de individuos arbustivos aislados con una cobertura del $5 \%$ compuesta por Duranta mutisii, Acacia decurrens y Baccharis latifolia, en los alrededores se encuentran frentes de avance de Ulex europaeus y poblaciones de Acacia decurrens. El mantenimiento en el área se llevó a cabo 30 días después de la intervención con el rastrillado de la hojarasca además de la plantación de especies nativas (350 individuos/0,5 Ha).

La evaluación de la regeneración de Acacia decurrens, Acacia melanoxylon y Ulex europaeus en cada área se realizó trimestralmente durante 18 meses, seleccionando por área una parcela permanente de $10 \times 10 \mathrm{~m}\left(100 \mathrm{~m}^{2}\right)$, para poder hacer el seguimiento del efecto del mantenimiento de control de las especies tensionantes y los cambios generados por el proceso de restauración ecológica, seleccionando aleatoriamente 10 cuadrantes de $1 \times 1 \mathrm{~m}$, en los que se realizó el levantamiento de información de presencia/ausencia, cobertura y estrato ocupado por los individuos de las especies evaluadas. Con la información obtenida se calculó la frecuencia relativa $\left(F R=F_{s p i} / F_{s p p t}\right)$, donde $F_{\mathrm{spi}}$ es la frecuencia de aparición de la especie, $F_{\mathrm{sppt}}$ es la frecuencia total de las especies, la cobertura relativa $\left(C R=\mathrm{C}_{\text {spi }} / \mathrm{C}_{\text {sppt }}\right)$, donde $\mathrm{C}_{\mathrm{spi}}$ es la cobertura de la especie $\mathrm{C}_{\mathrm{sppt}}$ es la cobertura total de las especies. 
Dos factores determinantes del proceso sucesional, corresponden a la oferta de espacios de suelo descubierto y la formación de la capa de hojarasca de foliolos de acacia, los cuales fueron evaluados a partir del registro de la cobertura en cada área, en cada uno de los cuadrantes seleccionados (10). Las diferencias entre las coberturas de las especies fueron comparadas con ANOVA de un factor, previa comprobación del supuesto de normalidad y homogeneidad de varianzas. Para el análisis del proceso de restauración ecológica se levantó la información de composición y riqueza de especies presentes en las tres parcelas permanentes de $100 \mathrm{~m}^{2}$ evaluadas, para el cálculo de la riqueza, diversidad de Shannon Wiener, equitatividad de Pielou y similaridad de Sorensen (Moreno, 2001).

\section{RESULTADOS Y DISCUSIÓN}

En el área intervenida 1, la dinámica de regeneración de las especies invasoras (Figura 1), registró la expresión del banco de semillas y rebrotes vegetativos de Acacia decurrens, alcanzando frecuencias de aparición superiores al $80 \%$, con una cobertura inferior al $1 \%$ en los primeros 90 días, posteriormente incrementa su presencia en la totalidad del área registrándose un aumento progresivo de la cobertura hasta alcanzar el $40 \%$ al final de la evaluación. Los individuos de Acacia melanoxylon presentaron baja frecuencia y cobertura durante el tiempo de evaluación, inferiores al $10 \%$. La producción de semillas de Acacia decurrens y Acacia melanoxylon es depositada en el suelo alimentando el banco de semillas persistente (Montenegro \& Vargas 2005; IAvH, 2003), sumado a la formación de un manto de hojarasca (Quiceno \& Medina 2006; DAMA \& BACHAQUEROS, 2000). Otros factores que inciden en la activación del banco de semillas de las especies tensionantes son la remoción del horizonte superficial del suelo y la presencia de incendios forestales (Bañalres et al. s.f.; Vargas, 2006).

Tal como se reportó en estudios anteriores en la misma zona, la frecuencia de aparición de Ulex europaeus en los primeros 90 días es total (Ríos, 2001), decreciendo a los 180 días, efecto de la eliminación de los renuevos de esta especie, con una cobertura menor al $5 \%$. Al final del monitoreo la frecuencia de aparición de los individuos de Ulex europaeus es superior al $90 \%$ con cobertura inferior al $10 \%$. La regeneración se presenta en áreas de claro donde aumenta el acceso de luz directa sobre el suelo activando el banco de semillas y el desarrollo de plántulas, que pueden ser inhibidas por la cobertura de las especies arbóreas (Muñoz, 2009), la vegetación herbácea y la rápida regeneración de los individuos de Acacia decurrens y Acacia melanoxylon.

La remoción de las especies invasoras y la hojarasca abre espacios de suelo superior al $80 \%$ de la cobertura, distribuidos por toda el área, los cuales van desapareciendo progresivamente por la acumulación de hojarasca, la regeneración de las especies tensionantes y colonización de especies. La defoliación y caída de vainas de los individuos adultos de acacia forman un nuevo manto de hojarasca que en los primeros 90 días tiene presencia en la totalidad del área con una cobertura del $62 \%$, la cual disminuye a los 180 días producto del mantenimiento realizado, permanenciendo al final de los 540 días del monitoreo con cobertura inferior al $40 \%$ (figura 1). La remoción del manto de hojarasca, activa los mecanismos de regeneración natural de las especies, permitiendo el arribo de propágulos, generando microhábitats que toleran el establecimiento y colonización de diversas especies como Digitalis purpurea, Phytolacca bogotensis, Senecio madagascariensis, solanum nigrum, Alonsoa meridionalis, Miconia squamulosa, Rubus bogotensis, Pteridium aquilinum, Passiflora mollisima, Cirsium vulgare, Anthoxanthum odoratum y Pennisetum clandestinum, entre las mas 
representativas, activando el proceso de sucesión secundaria (Martínez \& García, 2007).
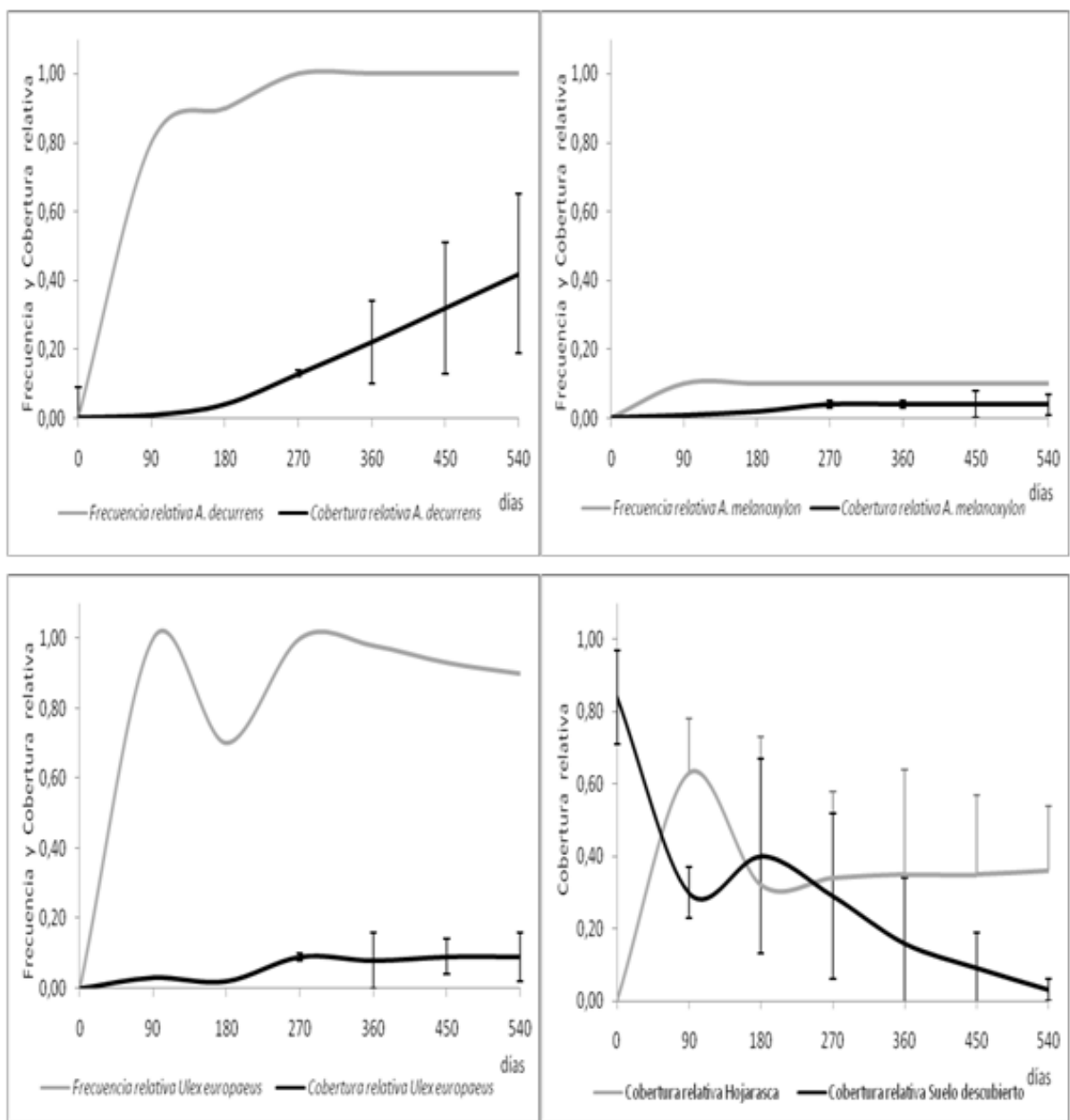

FIGURA 1. Frecuencia y cobertura relativa de $A$. decurrens y $A$. melanoxylon, $U$. europaeus, cobertura relativa de hojarasca y suelo descubierto, en el área intervenida 1.

En el área intervenida 2, la dinámica de regeneración de las especies invasoras (Figura 2), registró una activación del banco de semillas de Acacia decurrens distribuidas en el estrato rasante con coberturas inferiores al 10\%, mientras que los individuos de Acacia melanoxylon, presentaron baja presencia y cobertura, con mortalidad total a los 270 días, producto de la acumulación de hojarasca y disminución de la incidencia lumínica. Un patrón similar al de Acacia decurrens se evidencia en la regeneración de Ulex europaeus, con activación del banco de semillas en toda el área e incremento de la cobertura hasta el $20 \%$ en los primeros 90 días, posteriormente registra una frecuencia de aparición del $50 \%$ en el área y cobertura inferior al $10 \%$ al final del monitoreo (tabla 1). La hojarasca y el suelo descubierto, presentan una dinámica inversa, determinada por la defoliación de los individuos adultos de Acacia decurrens y Acacia melanoxylon (Figura 2). 

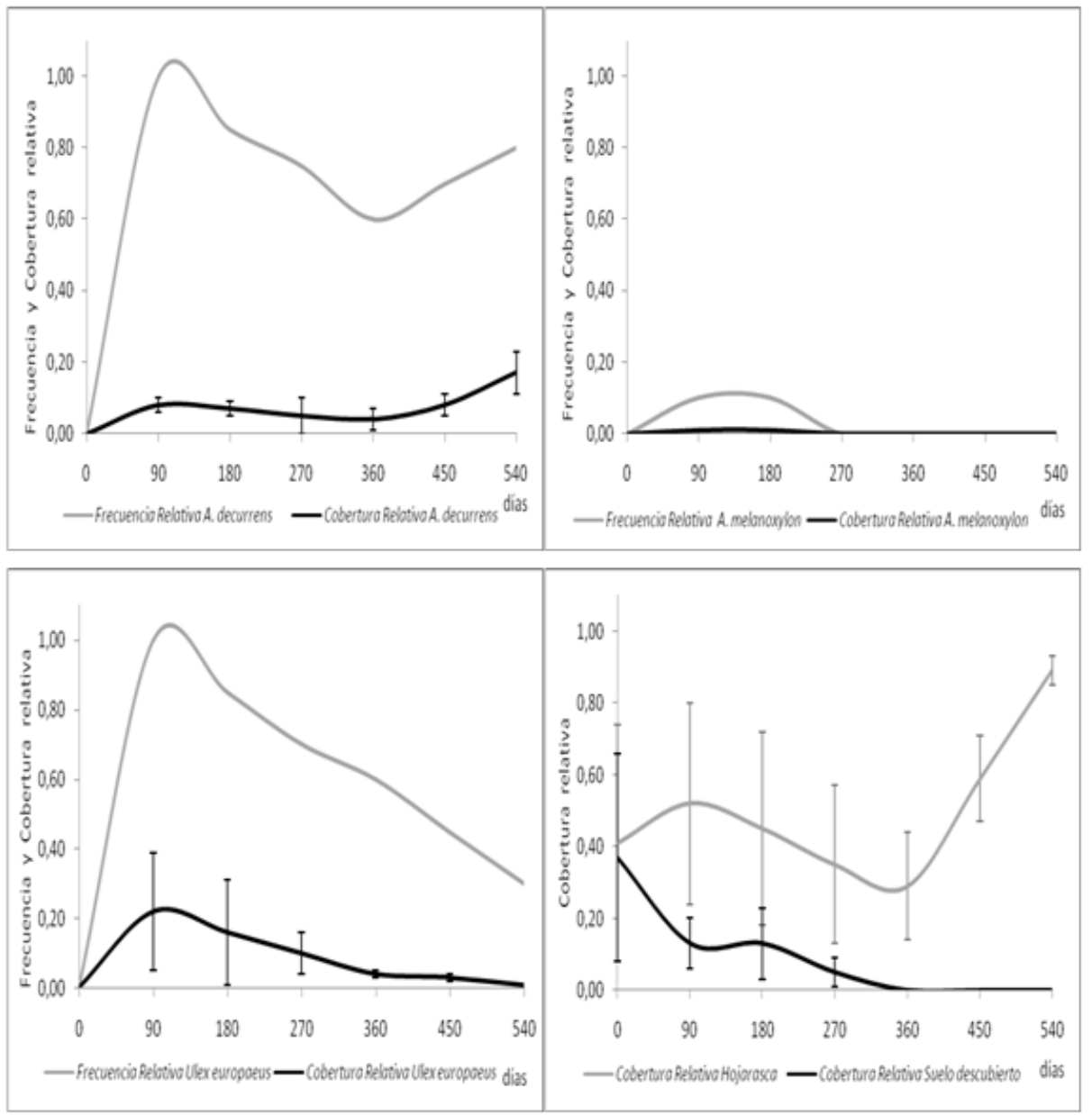

FIGURA 2. Frecuencia y cobertura relativa de $A$. decurrens y $A$. melanoxylon, $U$. europaeus, cobertura relativa de hojarasca y suelo descubierto, en el área intervenida 2.

La intensidad lumínica en las áreas está regulada por la presencia de individuos de porte arbustivo y arbóreo, que direccionan la dinámica de regeneración de acuerdo a su cobertura. Las coberturas densas con poca entrada de luz restringen el establecimiento de una amplia variedad de especies que se encuentran limitadas por la falta del recurso lumínico (Martínez \& García 2007; Acevedo et al. 2001), facilitando la progresiva acumulación de hojarasca y formación de abundante banco de semillas. Cuando la cobertura arbustiva y arbórea no permite la entrada de luz en el área se establecen especies esciófilas, que se encuentran adaptadas a condiciones limitadas de luz, encargándose de formar una cobertura herbácea y arbustiva en el sotobosque, que va determinando la composición y estructura del ecosistema. En esta área se ve favorecida la regeneración de Acacia decurrens y Acacia melanoxylon, con la formación del manto de hojarasca por presencia de individuos adultos de tales especies tensionantes. Los espacios de suelo descubierto son ocupados por el manto de hojarasca, inhibiendo la expresión del banco de semillas, el arribo y establecimiento de las especies nativas (Souto et al. 1993).

En el área intervenida 3, la dinámica de regeneración de las especies invasoras (Figura 3), registró una activación del banco de semillas de Acacia decurrens, con frecuencia de aparición que se incrementa progresivamente hasta el $60 \%$ del área, mientras que la cobertura de los 
individuos no supera el 5\%,, a su vez los individuos de Acacia melanoxylon tienen baja presencia y coberturas inferiores al $10 \%$.

La regeneración del banco de semillas de Ulex euroapeus es muy activo y rápidamente tiene presencia en toda el área, gradualmente aumenta la cobertura a medida que los individuos van creciendo, al cabo de los 540 días (tabla 1) se presenta una cobertura cercana al $60 \%$ del tota del área. Las condiciones estructurales del área favorecen el aumento de frecuencia y cobertura de los individuos de Ulex europaeus, siendo la especie con mayor tasa de regeneración entre las especies tensionantes evaluadas. La proporción de especies nativas y exóticas se va equilibrando gradualmente, por el arribo y persistencia de especies herbáceas ruderales que colonizan fácilmente los espacios de suelo descubierto con alta incidencia de luz solar, adaptándose fácilmente a condiciones de estrés hídrico y nutricional.

La cobertura de hojarasca presenta un leve incremento después de la intervención y dada la rápida colonización de especies va decreciendo hasta desaparecer. La oferta de suelo descubierto después de la intervención se extiende por toda el área, pero los espacios son rápidamente colonizados por especies de alta tasa de regeneración como Ulex europaeus, Digitalis purpurea, Anthoxanthum odoratum, Pteridium aquilinum y Rubus bogotensis.

En las áreas de claro con oferta lumínica durante todo el día, se observa la regeneración de especies heliófilas como Ulex europaeus $(20 \%$ cobertura), Digitalis purpurea (19\% de cobertura), Rubus bogotensis ( $8 \%$ cobertura) y Pteridium aquilinum (5\% de cobertura) principalmente, que utilizan mecanismos de propagación reproductivo y vegetativo que les permite ocupar los micrositios de implantación en el suelo luego de cortos períodos de tiempo, generando una fuerte competencia entre sí por la dominancia en el área. Dichas especies se adaptan a las condiciones de suelos degradados con escases de nutrientes y baja humedad (Holmberg et al. 2007), limitando el establecimiento de especies de porte arbustivo y arbóreo. En estas áreas se ve favorecida la regeneración de Ulex europaeus que activa el banco de semillas con producción abundante de plántulas que van generando un manto que cubre rapidamente el estrato herbáceo (Torres 2009, DAMA \& BACHAQUEROS, 2000). empiezando a competir por recursos y espacio con especies pioneras de rápido crecimiento como Pennisetum clandestinum, Anthoxanthum odoratum, Hypochaeris radicata, Pteridium aquilinum, Digitalis purpurea y Rubus bogotensis, desapareciendo los espacios de suelo descubierto. 

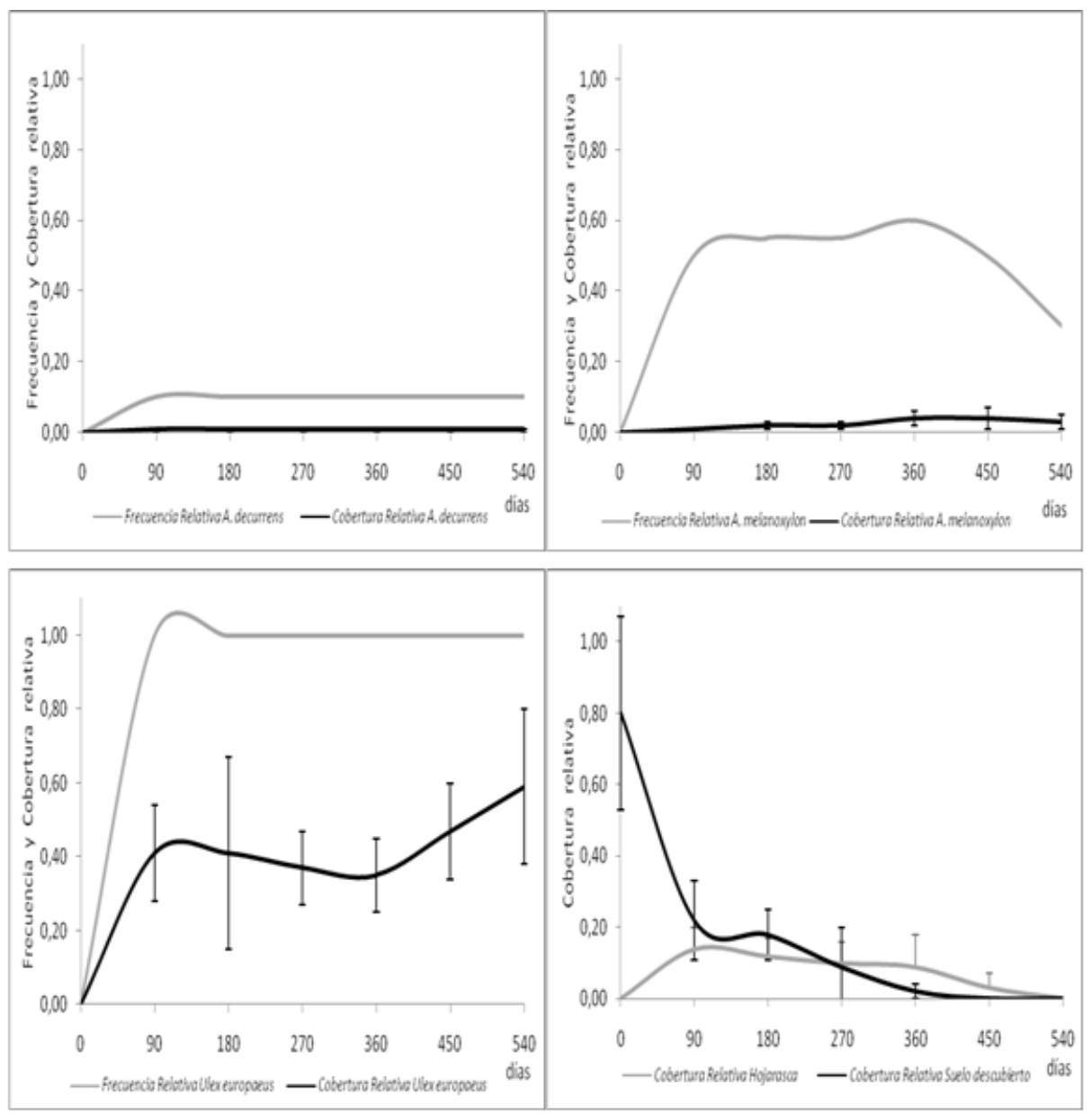

FIGURA 3. Frecuencia y cobertura relativa de $A$. decurrens y $A$. melanoxylon, $U$. europaeus, cobertura relativa de hojarasca y suelo descubierto, en el área intervenida 3.

Al cabo de 540 días, la cobertura de los individuos de Acacia decurrens no presentan diferencias significativas $\left(F_{2,27}=3,23 ; p>0,05\right)$ entre las tres áreas en proceso de restauración ecológica evaluadas, no obstante si hay diferencias significativas $\left(F_{1,18}=7,18 ; p<0,05\right)$ entre la cobertura del área intervenida 1 con una cobertura del $30,5 \%$ y el área intervenida 3 con una cobertura del 1\%. La cobertura de los individuos de Acacia melanoxylon no presenta diferencias significativas en las tres áreas $\left(F_{2,27}=2,57 ; p>0,05\right)$ por la baja regeneración de la especie en la zona. Por el contrario, la comparación de coberturas de los individuos de Ulex europaeus si presentan diferencias significativas entre las tres áreas evaluadas $\left(F_{2,27}=51,75 ; p<0,05\right)$, debido a la rápida colonización del área intervenida 3 con una cobertura del $59 \%$, con respecto al $8,5 \%$ del área intervenida $1\left(\mathrm{~F}_{1,18}=41,38 ; \mathrm{p}<0,05\right)$ y el $0,5 \%$ del área intervenida 2 $\left(F_{1,18}=63,59 ; p<0,05\right)$.

La invasión de las especies exóticas en áreas degradadas generan cambios en la dinámica sucesional de acuerdo a la magnitud espacial y temporal de la alteración (Martínez \& García, 2007; Vila et al. 2006; DAMA \& SUNA HISCA, 2002). Las coberturas de Acacia decurrens se encuentran sucesionalmente detenidas, por la densa cobertura arbórea y la formación del manto de hojarasca que inhibe la expresión del banco de semillas, limita la oferta de recursos relacionado con el aporte de 
materia orgánica, que ha sido reportado por Bolívar et al. (2001) que encontró bajo contenido de bases en las hojas de acacia y la hojarasca con altos porcentajes de lignina y taninos indican una lenta descomposición de la materia orgànica, bajo reciclaje de nutrientes además de cambio en las condiciones edáficas disminuyendo el $\mathrm{pH}$, lo que en conjunto representa un riesgo para la iniciación y propagación de incendios forestales (DAMA \& SUNA HISCA, 2002; DAMA \& BACHAQUEROS, 2000). La exclusión de especies nativas no permite que los procesos funcionales del ecosistema se dimamicen y éste entra en un período de latencia.

La evaluación de la riqueza y la diversidad en las tres áreas intervenidas, muestra con respecto al área blanco un incremento gradual durante el proceso de sucesión secundaria. Del igual modo, hay un incremento en la equitatividad de las especies presentes en el área de las tres áreas durante la primera etapa de establecimiento, iniciando procesos de competencia, la activación de mecanismos, estrategias de adaptación y respuesta ante los diferentes niveles de estrés, conformando comunidades vegetales diversas, estratificadas, con una oferta de hábitat y nichos de especies de artrópodos además de aves, dinamizando los porcesos ecológicos de conectividad entre áreas conservadas. Las áreas intervenidas presentan similaridad inferior al $30 \%$ con respecto al área blanco y mantienen una similaridad entre ellas superior al $50 \%$, relacionándose de acuerdo al tiempo de avance del proceso de restauración ecológica, el tipo de tratamiento aplicado y las condiciones estructurales del área, lo cual evidencia el progreso del proceso de restauración ecológica en la dinámica de conformación de nuevas comunidades vegetales (Figura 4).

La incorporación de especies nativas (Juglans neotropica, Quercus humboldtii, Myrcianthes leucoxyla, Tibouchina mollis, Aegiphilla bogotensis, Abatia parviflora, Dodonaea viscosa, Ageratina aristeii y Baccharis latifolia), que se establecieron en el área y se convierten en un factor fundamental para el control de especies tensionantes en las áreas de claro, ya que, una vez alcanzan el estrato arbustivo las especies de rápido crecimiento (Aegiphilla bogotensis, Abatia parviflora y Bacharis latifolia, entre los 360 y 540 días) o generan coberturas amplias de copa (Aegiphilla bogotensis mayor $3000 \mathrm{~cm}^{2}$, Baccharis latifolia mayor a $3500 \mathrm{~cm}^{2}$ y el resto de especies reintroducidas con coberturas entre los 1500 y $2500 \mathrm{~cm}^{2}$ ), que empiezan a producir cambios en la condiciones microclimáticas (Padilla 2008; DAMA \& BACHAQUEROS, 2000), inhibiendo el crecimiento de las especies heliófilas, facilitando el arribo de especies nativas silvestres (Quiroz, C. et al. 2009), creando una barrera para la acumulación de hojarasca de acacia. La hojarasca producida por las especies nativas plantadas es de rápida descomposición y acumulación de humedad, que minimiza el riesgo de iniciación o propagación de incendios forestales; aportando materia orgánica al suelo con aumento de los micronutrientes y cambios en las condiciones físicas del mismo (Bonilla et al. 2008), esenciales para el sostenimiento de la estructura y función de la biota y recuperación de las condiones naturales del área.

Dentro del proceso de restauración ecológica, las especies que colonizaron o fueron reintroducidas durante los 540 días de monitoreo, fueron: Acacia decurrens, Acacia melanoxylon, Ulex europaeus, Leptodontium sp, Aegiphilla bogotensis, Myrcianthes leucoxyla, Dodonaea viscosa, Ageratina aristeii, Duranta mutisii, Quercus humboldtii, Juglans neotropica, Rubus bogotensis, Conyza cf. floribundum, Solanum nigrum, Digitalis purpurea, Alonsoa meridionalis, Oxalis cf. spiralis, Phytolacca bogotensis, Abatia parviflora, Dodonaea viscosa, Bacharis latifolia, Gnaphalium sp, Pennisetum clandestinum, Senecio madagascariensis, Crocosmia $x$ crocosmiflora, Baccharis latifolia, Cirsium vulgare, Citharexylum subflavecens, Miconia 
squamulosa, Tradescantia sp. Anthoxanthum odoratum, Lepichinia sp, Hypochaeris radicata, Taraxacum officinale, Miconia ligustrina, Muehlenbeckia tamnifolia, Agrostis sp., Pennisetum clandestinum, Cucurbita sp., Passiflora mollisima, Prunus serotina, Pteridium aquilinum, Tibouchina mollis, de las cuales al final de la evaluación no se registró presencia de Solanum nigrum, Gnaphalium sp, Miconia ligustrina, Tradescantia sp, Lepichinia sp, Taraxacum officinale, Cucurbita sp. Passiflora mollisima y Prunus serotina.
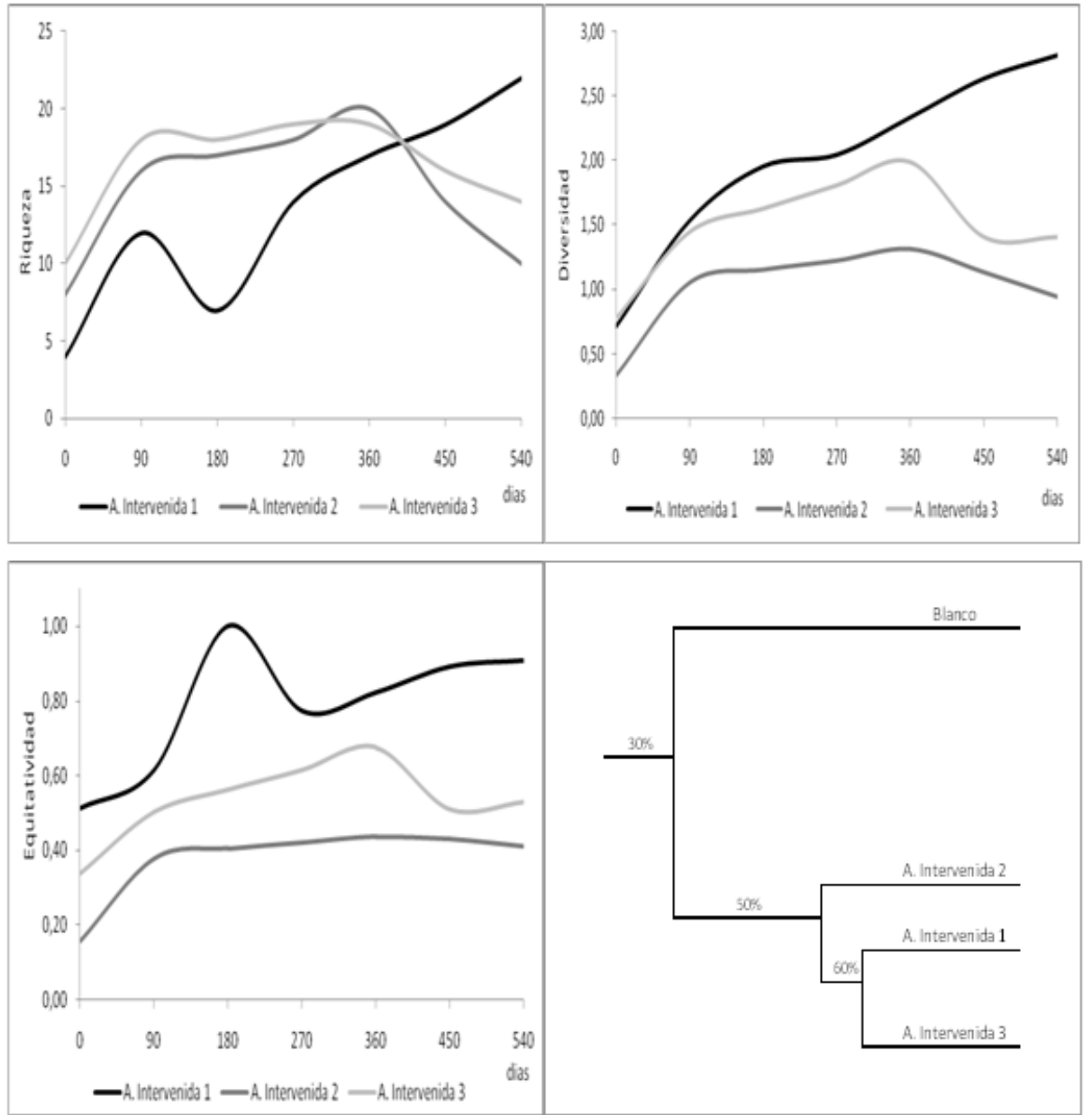

FIGURA 4. Riqueza de especies, diversidad de Shannon Wiener, equitatividad de Pielou y similaridad de Sorensen, en el área blanco (monocultivo de Acacia decurrens) y las tres áreas intervenidas, al cabo de 540 días de seguimiento al proceso de restauración ecológica.

Los mantenimientos de los renuevos, con la remoción de plántulas y brotes vegetativos de Acacia decurrens, Acacia melanoxylon y Ulex europaeus, son primordiales dentro del proceso de rehabilitación del área, ya que, permiten dar control a las especies tensionantes, limitar la acumulación de hojarasca y aumentar la oferta de espacio de suelo descubierto que permite el arribo de propágulos y el establecimiento de nuevas especies que aumentan la riqueza sin presentar diferencias significativas entre los tratamientos $\left(F_{2,24}=0,45 ; p<0,05\right)$, diversidad con diferencias significativas entre los tratamientos $\left(F_{2,24}=4,47 ; p<0,05\right)$ y equitativiadad del área, garantizando la dinámica sucesional propia de los estadios iniciales del proceso, con el arribo de especies pioneras de rápido crecimiento que generan cobertura en el estrato rasante del suelo y facilitan la llegada de la fauna asociada, aportando materia orgánica 
que potencie el reciclaje de nutrientes, para el posterior arribo y colonización de especies leñosas nativas tempranas y tardías.

\section{CONCLUSIONES}

La dinámica de regeneración para las especies invasoras en el proceso de restauración ecológica, está determinada por la expresión del banco de semillas y las estructuras vegetativas remanentes.

El mantenimiento de control respecto a la regeneración de las especies invasoras durante el proceso de restauración ecológica, limita la capacidad invasora en la especie y mantiene la oferta de micrositios para la colonización de especies nativas.

En las áreas con mayor acumulación de hojarasca de Acacia decurrens y Acacia melanoxylon, se evidencia menor cobertura de especies regeneradas.

El proceso de restauración ecológica activa los mecanismos sucesionales, evidenciado en el incremento de la riqueza y diversidad de especies vegetales con respecto al monocultivo de acacia, generando una dinámica de colonización con especies ruderales de rápido crecimiento, además del establecimiento de las especies nativas plantadas, lo cual, disminuye la similaridad (con un patrón constante desde el momento de la implementación del proceso de restauración ecológica) de las áreas intervenidas con respecto al área blanco, la cual presenta una etapa de sucesión detenida con alto riesgo para la generación y propagación de incendios forestales.

\section{Agradecimientos}

La presente investigación ha sido posible gracias al apoyo del equipo profesional, técnico y operativo del proyecto de restauración ecológica del Jardín Botánico José Celestino Mutis. Agradezco al Biólogo Héctor Felipe Ríos, por las observaciones hechas al documento.

\section{BIBLIOGRAFÍA}

- Acevedo, M., S. Monteleone, M. Ataroff \& C. Estrada. (2001). Aberturas de dosel y espectro de la luz en el Sotobosque de una Selva Nublada Andina de Venezuela. Ciencia. 9(2): 165-183.

- Aguilar, M. (2010). Restauración ecológica de áreas afectadas por Ulex europaeus I. Serranía El Zuque, Reserva Forestal Bosque Oriental de Bogotá, localidad 4 San Cristóbal, Bogotá D. C., Colombia. Tesis de Maestría en Restauración de Ecosistemas. Universidad de Alcalá: 71.

- Bañalres, P., M. Sarasola, H. Vera, M. Arim, M. Clara, E. Grosso \& V. Korenko. s.f. Diagnóstico de los bosques costeros del Uruguay: 1-16. Mvotma - Universidad de la República. Uruguay.

- Bolívar, D., M. Ibrahim \& D. Kass. (2001). Características químicas de un suelo ácido y composición mineral de Brachiaria humidicola bajo un sistema pastoril con Acacia mangium. Costa Rica: 15. 
- Bonilla, R., B. Roncallo, J. Jimeno \& T. García. (2008). Producción y descomposición de la hojarasca en bosque nativos y de Leucaena sp, en Codazzi, Cesar. Rev. Corpoica. 9(2): 5-11.

- DAMA \& Fundación Estación Biológica Bachaqueros. (2000). Protocolo Distrital de Restauración Ecológica: Guía para la restauración de ecosistemas nativos en las áreas rurales de Santa Fe de Bogotá: 276. Secretaria Distrital de Ambiente. Bogotá.

- DAMA \& SUNA HISCA. (2002). Parque Ecológico Distrital de Montaña Entrenubes. Componente biofísico: 473. Bogotá.

- Díaz, A. \& O. Vargas. (2009). Efecto sobre la siembra de leguminosas herbáceas y arbustivas sobre el control en el establecimiento de la especie invasora Ulex europaeus L. (fabaceae) en los Alrededores de Chisacá, Localidad de Usme. En: Libro de resúmenes, I Congreso Colombiano de Restauración Ecológica: 275. Grupo de Restauración Ecológica de la Universidad Nacional de Colombia.

- Funes, G., S. Diaz \& P. Venier. (2009). La temperatura como principal determinante de la germinación en especies del Chaco Seco del Argentina. Ecología Austral. 19: 129-138.

- Galvis, J. (2002). La restauración ecológica: conceptos y aplicaciones. Universidad Rafael Landivar: 22.

- García R., A. Pauchard, L. Cavieres, E. Peña \& M. Rodríguez. (2010). El fuego favorece la invasión de Teline monspessulana (Fabaceae) al aumentar su germinación. Revista Chilena de Historia Natural. 83: 443452.

- Gómez, S. (2008). Patrones y mecanismos de regeneración por semillas tras el fuego en el matorral mediterráneo de Chile Central: consecuencias en la invasión de especies alóctonas. Trabajo de grado. Universidad de Concepción, Chile: 113.

- Holmberg, G., E. Siebald R. De La Barra, \& D. Duboid. (2007). Estrategia para el control del Espinillo. Instituto de Investigaciones Agropecuarias Centro Regional de Investigación Remehue. Chile. Informativo. 58: 1-4.

- Instituto de Investigación de Recursos Biológicos Alexander von Humboldt -IAvH-. (2003). Estudio del mercado colombiano de semillas forestales: 101. Bogotá.

- Leython, S \& T. Ruiz. (2006). Leguminosae de un bosque estacional, La Trilla, Parque Nacional Henri Pittier, Estado Aragua, Venezuela. Ernstia. 16 (2): 81-94.

- Mack, R., D. Simberloff \& M. Lonsdale. (2000). Invasiones biológicas: causas, epidemiología, consecuencias globales y control. Tópicos en Ecología. 5: 1-19.

- Matthews, S. (2005). El programa Mundial sobre Especies Invasoras. Secretaría GISP: 80.

- Martínez, M. \& X. García. (2007). Sucesión ecológica y restauración de las selvas húmedas. Bol. Soc. Bot. Méx. 80: 69-84.

- Medina, M. H. Orozco \& C. Díez. (2008). Establecimiento de un sistema silvopastoril mediante las especies Alnus acuminata H.B.K. y Acacia decurrens Willd y respuesta al empleo de organismos rizosféricos en San Pedro (Antioquia). Livestock Research for Rural Development: 20 (7).

- Montenegro, A. \& O. Vargas. (2005). Estrategias de regeneración del banco de semillas en una comunidad de bosque altoandino secundario. En: Bonilla, A. (Ed) Estrategias adaptativas de Plantas del Páramo y del Bosque Altoandino en la Cordillera Oriental de Colombia: 353 Universidad Nacional de Colombia. Bogotá.

- Moreno, C. (2001). Métodos para medir la biodiversidad. M \& T Manuales y Tesis SEA: 84 Zaragoza, España.

- Muñoz, E. (2009). Control biológico de zonas invadidas por Ulex europaeus L. (Magnoliophyta: Fabaceae) mediante sustitución por vegetación nativa en la Isla del Rey, región de los Ríos, Chile. Gestión Ambiental 18:11-30. 
- Padilla, F. (2008). Factores limitantes y estrategias de establecimiento de plantas leñosas en ambientes semiáridos. Implicaciones para la restauración. Ecosistemas. 17(1):155 - 159.

- Quiceno, M. \& M. Medina. (2006). La Acacia decurrens Will fuente potencial de biomasa nutritiva para la ganadería del trópico de altura. Livestock Research for Rural Development. 18(12).

- Quiroz, C., A. Pauchard, A. Marticorena \& L. Cavieres. (2009). Manual de plantas invasoras del centro sur de Chile: 45. Chile.

- Quiroz, I., M. González, E. García, C. Casanova \& H. Soto. (2009). Ensayo de germinación para semillas de Acacia mearnsii De Wild procedentes de la zona de Valdivia, Región de Los Ríos. Instituto Forestal - INFOR, Centro Tecnológico de la Planta Forestal - CTPF: 8. Concepción, Chile.

- Ríos A., H. F. (2001). Eliminación de la especie invasora Ulex europaeus L (fabaceae) como estrategia experimental de restauración de la vegetación en el Cerro de Monserrate, Bogotá D. C, Colombia. Trabajo de Grado para optar al título de biólogo. Universidad Nacional de Colombia. Facultad de Ciencias. Departamento de Biología. Bogotá D. C.

- Ríos A., H. F. (2010). Acumulación de fitomasa y bancos de semillas in invasiones de retamo espinoso (Ulex europaeus $\mathrm{L}$ ) en diferentes estados de desarrollo en la cuenca del río Teusacá, Bogotá D.C., Colombia. Trabajo de Grado. Universidad Distrital Francisco José de Caldas. Facultad de Medio Ambiente y Recursos Naturales. Especialización en Gerencia de Recursos Naturales.

- Rodríguez, S. (2009). Organismos del Suelo: La dimensión invisible de las invasiones por plantas no nativas. Ecosistemas. 18 (2):32 - 43.

- Sánchez, A. (2009). Ciclos de retroalimentación positiva en la invasión de retamo espinoso (Ulex europaeus) en los andes colombianos. En: Libro de resúmenes, I Congreso Colombiano de Restauración Ecológica. Grupo de Restauración Ecológica de la Universidad Nacional de Colombia.

- Santana, M. (2007). Fijación biológica de nitrógeno por leguminosas arbóreas para sombra de café en Puerto Rico. 91. Tesis de grado maestría en Agronomía. Universidad de Puerto Rico. Mayagüe.

- Souto, X., L. González \& M. Reigosa. (1993). Estudios de los efectos alelopáticos producidos por partes aéreas de distintas especies arbóreas (Eucalyptus globulus, Acacia melanoxylon, Quercus rubor, Pinus radicata) en descomposición en el suelo: 189 - 194. Congreso Forestal Español, España.

- Torres, N. (2009). Banco de semillas germinable en áreas invadidas por retamo espinoso (Ulex europaeus) con diferentes edades de quema (alrededores del Embalse de Chisacá Bogotá- Localidad de Usme). Trabajo de Grado para Optar al Título de Biólogo: 110. Universidad Militar Nueva Granada. Bogotá.

- Vargas, O.(2009). Estrategias para la restauración ecológica del bosque altoandino. El caso de la Reserva Forestal Municipal de Cogua, Cundinamarca. Universidad Nacional de Colombia: 369.

- Vargas, O. (2006). En búsqueda del bosque perdido, una experiencia de restauración ecológica en predios del Embalse de Chisacá: 115. Grupo de Restauración Ecológica, Universidad Nacional de Colombia.

- Vila, M., S. Bacher, P. Hulme, M. Kenis, M. Kobelt, W. Nentwig, D. Sol, \& W. Solarz. (2006). Impactos ecológicos de las invasiones de plantas y vertebrados terrestres en Europa. Ecosistemas.15 (2):13 - 23.

- Zabaleta, A. \& O. Vargas. (2009). Expresión in situ del banco de semillas germinable de Ulex europaeus y su relación con la estructura de los matorrales. En: Vargas, O. Estrategias para la restauración ecológica del bosque altoandino. El caso de la Reserva Forestal Municipal de Cogua, Cundinamarca: 372. Universidad Nacional de Colombia. Bogotá. 
1. Biólogo. Línea de investigación en evaluación y seguimiento a procesos de restauración ecológica de áreas alteradas del Distrito Capital y la Región. Subdirección Científica. Jardín Botánico José Celestino Mutis. Correo electrónico: jhsolorzab@gmail.com 